\title{
The ubiquity of conservative translations
}

\author{
Emil Jeřábek* \\ Institute of Mathematics of the Academy of Sciences \\ Žitná 25, 11567 Praha 1, Czech Republic, email: jerabek@math.cas.cz
}

October 16, 2018

\begin{abstract}
We study the notion of conservative translation between logics introduced by Feitosa and D'Ottaviano [7]. We show that classical propositional logic (CPC) is universal in the sense that every finitary consequence relation over a countable set of formulas can be conservatively translated into CPC. The translation is computable if the consequence relation is decidable. More generally, we show that one can take instead of CPC a broad class of logics (extensions of a certain fragment of full Lambek calculus FL) including most nonclassical logics studied in the literature, hence in a sense, (almost) any two reasonable deductive systems can be conservatively translated into each other. We also provide some counterexamples, in particular the paraconsistent logic LP is not universal.
\end{abstract}

\section{Introduction}

There have been several proposals of a general concept of a translation or interpretation between abstract logical systems, see e.g. [1, 10] for overviews. A minimalist approach was taken by da Silva, D'Ottaviano and Sette [13]: a logic (deductive system) is given by any Tarski-style consequence operator, and then a translation of one logic in another is an arbitrary mapping of formulas to formulas preserving the consequence relation. Feitosa and D'Ottaviano [7] consider the stronger notion of conservative translations, which preserve the consequence relation in both directions. This avoids uninteresting examples of translations such as mapping all formulas to a fixed tautology.

This notion of a conservative translation is still very general (perhaps too general): for instance, translations are not required to respect the structure of formulas in any way, to be computable, or to preserve any properties of the logic. For this reason, it is natural to expect that there should exist a conservative translation between more or less any two reasonable deductive systems. Nevertheless, no result to such effect appears in the literature. Instead, there are several papers presenting proofs (often non-constructive) of the existence of conservative translations between particular pairs of logics: $[3,4,5,6]$.

\footnotetext{
* Supported by grant IAA100190902 of GA AV ČR, project 1M0545 of MŠMT ČR, RVO: 67985840, and a grant from the John Templeton Foundation.
} 
The main purpose of this paper is to demonstrate that for a quite large class of logics, it is indeed possible to construct a conservative translation between any two of them. First, we prove that an arbitrary finitary deductive system $L$ in countably many formulas can be conservatively translated into classical propositional logic (CPC), in either the single-conclusion or multiple-conclusion setting. Our translation is constructed by an explicit inductive definition, and it is computable whenever $L$ is decidable. Moreover, the translation has the additional property of being most general in the sense that every other translation of $L$ to CPC is equivalent to its substitution instance.

Let us define a logic $L$ to be universal if every finitary deductive system in countably many formulas can be conservatively translated into $L$, so that the result above can be restated by saying that CPC is universal. We generalize this result by showing that every deductive system between the $\rightarrow, \leftarrow, \wedge$ fragment of the full Lambek calculus FL (see [8]) and the

corresponding fragment of CPC is universal, and similarly, any deductive system between BCK (the implication fragment of $\mathbf{F L}_{\text {ew }}$ ) and $\mathbf{C P C} \uparrow \rightarrow$ is universal. This establishes the universality of most of nonclassical logics studied in the literature, as they typically extend (a suitable fragment of) FL in one way or another: this includes e.g. intuitionistic and intermediate logics, various modal, substructural, fuzzy, or relevant logics, both propositional and first-order.

As an additional example, we show that Kleene's logic with truth constants is universal, whereas the paraconsistent logic $\mathbf{L P}$ - based on the same algebra but with a different choice of designated truth values - is not universal. We also completely characterize universal fragments of CPC: a fragment $\mathbf{C P C} \uparrow_{B}$ is universal if and only if implication is definable from $B$. (In particular, we obtain a couple of nontrivial examples of logics into which $\mathbf{C P C}$ cannot be conservatively translated, namely $\mathbf{L P}$ and the fragments $\mathbf{C P C} \uparrow_{\leftrightarrow, \neg}, \mathbf{C P C} \uparrow_{\wedge, \vee, \perp, T}$.)

The paper is organized as follows. In Section 2 we give basic definitions, and we construct conservative translations into classical logic. In Section 3 we investigate the class of universal deductive systems, as detailed above. Section 4 consists of concluding remarks.

\section{Translation to classical logic}

In this section, we are going to construct conservative translations of (almost) arbitrary logics into classical logic (Theorem 2.4). First we review the relevant definitions to fix the notation.

Definition 2.1 A pair $L=\langle F, \vdash\rangle$ is a deductive system (or logic) over a set of formulas $F$ if $\vdash \subseteq \mathcal{P}(F) \times F$ is a Tarski-style consequence relation, i.e., if it satisfies

(i) $\varphi \vdash \varphi$,

(ii) $\Gamma \vdash \varphi$ implies $\Gamma, \Gamma^{\prime} \vdash \varphi$,

(iii) if $\Gamma \vdash \varphi$ and $\Delta \vdash \psi$ for all $\psi \in \Gamma$, then $\Delta \vdash \varphi$,

for every $\varphi \in F$ and $\Gamma, \Gamma^{\prime}, \Delta \subseteq F$. A deductive system is finitary if

(iv) $\Gamma \vdash \varphi$ implies $\Gamma^{\prime} \vdash \varphi$ for some finite $\Gamma^{\prime} \subseteq \Gamma$. 
When discussing algorithmic issues, we will tacitly assume that $F$ is encoded as a recursively enumerable subset of $\omega$. If $X=\left\langle\varphi_{1}, \ldots, \varphi_{n}\right\rangle$ is a sequence of formulas, we will also write $X \vdash \varphi$ instead of $\left\{\varphi_{1}, \ldots, \varphi_{n}\right\} \vdash \varphi$ by abuse of notation (we will never use $\vdash$ for a sequent arrow).

A deductive system $L=\langle F, \vdash\rangle$ is a propositional logic if $F$ is the set of formulas built inductively from a set of variables and a set of finitary connectives (i.e., $F$ is a free algebra in a particular signature), and $\vdash$ is structural (substitution-invariant):

(v) $\Gamma \vdash \varphi$ implies $\sigma(\Gamma) \vdash \sigma(\varphi)$ for every substitution $\sigma$,

where as usual, a substitution is a homomorphism of free algebras. Let $\mathbf{C P C}=\left\langle F_{\mathbf{C P C}}, \vdash_{\mathbf{C P C}}\right\rangle$ denote the usual consequence relation of classical propositional logic in countably infinitely many variables using an arbitrary functionally complete finite set of Boolean connectives (the exact choice does not matter, as classical consequence is unaffected by translation of formulas to a language with a different set of basic connectives). In CPC, we will employ big conjunctions and disjunctions $\bigwedge \Gamma, \bigvee \Gamma$ in the usual way; in particular, $\bigwedge \varnothing=\top$ and $\bigvee \varnothing=\perp$ (again, it does not matter whether these constants are included in the set of basic connectives, or defined by equivalent more complicated formulas, even if they involve extra variables).

Definition 2.2 A translation from a deductive system $L_{0}=\left\langle F_{0}, \vdash_{0}\right\rangle$ to a deductive system $L_{1}=\left\langle F_{1}, \vdash_{1}\right\rangle$ is a function $f: F_{0} \rightarrow F_{1}$ such that

$$
\Gamma \vdash_{0} \varphi \Rightarrow f(\Gamma) \vdash_{1} f(\varphi)
$$

for every $\Gamma \subseteq F_{0}, \varphi \in F_{0}$. We will write this as $f: L_{0} \rightarrow L_{1}$. The translation $f$ is conservative, written as $f: L_{0} \rightarrow_{c} L_{1}$, if

$$
\Gamma \vdash_{0} \varphi \Leftrightarrow f(\Gamma) \vdash_{1} f(\varphi) .
$$

We write $L_{0} \leq_{c} L_{1}$ if there exists a conservative translation $f: L_{0} \rightarrow_{c} L_{1}$.

The translations to classical logic we construct have an additional property which might be of independent interest, hence we give it a name:

Definition 2.3 Let $L_{0}=\left\langle F_{0}, \vdash_{0}\right\rangle$ be a deductive system, and $L_{1}=\left\langle F_{1}, \vdash_{1}\right\rangle$ a propositional logic. A translation $f: L_{0} \rightarrow L_{1}$ is most general if for every translation $g: L_{0} \rightarrow L_{1}$, there exists a substitution $\sigma$ such that $g(\varphi) \vdash_{1} \sigma(f(\varphi))$ for every $\varphi \in F_{0}$.

Notice that if $L_{0} \leq_{c} L_{1}$ and $L_{1}$ is finitary, then $L_{0}$ is also finitary.

The main result of this section is:

Theorem 2.4 For every finitary deductive system $L=\langle F, \vdash\rangle$ over a countable set of formulas $F$, there exists a conservative most general translation $f: L \rightarrow_{c}$ CPC.

If $\vdash$ is decidable, then $f$ is computable. In general, $f$ is Turing equivalent to (the finitary fragment of $) \vdash$. 
We will prove Theorem 2.4 below as a corollary to its multiple-conclusion version. Apart from being more general, the construction of the translations in the multiple-conclusion case is more transparent and displays better the underlying symmetry, we thus find it preferable to giving a direct proof for the single-conclusion case, which feels a bit ad hoc.

Definition 2.5 A pair $L=\langle F, \vdash\rangle$ is a multiple-conclusion deductive system (or multipleconclusion logic) [12] if $\vdash \subseteq \mathcal{P}(F) \times \mathcal{P}(F)$ satisfies

(i) $\varphi \vdash \varphi$,

(ii) $\Gamma \vdash \Delta$ implies $\Gamma, \Gamma^{\prime} \vdash \Delta, \Delta^{\prime}$,

(iii) if $\Gamma, \Pi \vdash \Lambda, \Delta$ for every $\Pi, \Lambda$ such that $\Pi \cup \Lambda=\Xi$, then $\Gamma \vdash \Delta$,

for every $\varphi \in F$ and $\Gamma, \Gamma^{\prime}, \Delta, \Delta^{\prime}, \Xi \subseteq F$. (Condition (iii) is a form of the cut rule.) A multiple-conclusion deductive system is finitary if

(iv) $\Gamma \vdash \Delta$ implies $\Gamma^{\prime} \vdash \Delta^{\prime}$ for some finite $\Gamma^{\prime} \subseteq \Gamma, \Delta^{\prime} \subseteq \Delta$.

Note that if $L$ is finitary, condition (iii) can be equivalently simplified to

(iii') $^{\prime}$ if $\Gamma, \varphi \vdash \Delta$ and $\Gamma \vdash \varphi, \Delta$, then $\Gamma \vdash \Delta$.

$L$ is consistent if $\varnothing \nvdash \varnothing$. Let $\mathbf{C P C}_{m}=\left\langle F_{\mathbf{C P C}}, \vDash_{\mathbf{C P C}}\right\rangle$ denote the maximal structural multiple-conclusion consequence relation for classical propositional logic: $\Gamma \vDash_{\text {CPC }} \Delta$ iff there is no $0-1$ assignment $v$ such that $v(\varphi)=1$ for all $\varphi \in \Gamma$ and $v(\psi)=0$ for all $\psi \in \Delta$. (In other words, $\Gamma \vDash_{\mathbf{C P C}} \Delta$ iff there are finite subsets $\Gamma^{\prime} \subseteq \Gamma, \Delta^{\prime} \subseteq \Delta$ such that $\vdash_{\mathbf{C P C}} \wedge \Gamma^{\prime} \rightarrow \bigvee \Delta^{\prime}$.)

We generalize the notions of translations, conservative translations, propositional logics, and most general translations to the multiple-conclusion setting in the obvious way.

Theorem 2.6 For every finitary consistent multiple-conclusion deductive system $L=\langle F, \vdash\rangle$ over a countable set of formulas $F$, there exists a conservative most general translation $f: L \rightarrow_{c} \mathbf{C P C}_{m}$.

If $\vdash$ is decidable, then $f$ is computable. In general, $f$ is Turing equivalent to $\vdash$.

Proof: Let $F=\left\{\alpha_{n}: n \in \omega\right\}$ be a (not necessarily injective) enumeration. We will define a sequence of formulas $f\left(\alpha_{n}\right):=\beta_{n} \in F_{\mathbf{C P C}}$ by induction on $n$. We denote by $p_{n}$ the $n$th propositional variable of CPC. We abbreviate $\bar{\alpha}_{X}=\left\{\alpha_{i}: i \in X\right\}$, and similarly for $\bar{\beta}_{X}$; moreover, we are going to use the identity $n=\{i \in \omega: i<n\}$.

Assume by the induction hypothesis that $\beta_{i}$ have been already defined for all $i<n$ in such a way that

$$
\bar{\alpha}_{X} \vdash \bar{\alpha}_{Y} \Rightarrow \bar{\beta}_{X} \vDash_{\mathbf{C P C}} \bar{\beta}_{Y}
$$


for every $X, Y \subseteq n$. (This holds for $n=0$ as $\vdash$ is consistent by assumption.) Define ${ }^{1} \beta_{n}$ by

$$
\begin{aligned}
& \gamma_{n}:=\bigvee_{\substack{X, Y \subseteq n \\
\alpha_{X} \vdash \alpha_{n}, \bar{\alpha}_{Y}}}\left(\bigwedge \bar{\beta}_{X} \wedge \neg \bigvee \bar{\beta}_{Y}\right), \\
& \delta_{n}:= \bigwedge_{\substack{X, Y \subseteq n \\
\alpha_{X}, \alpha_{n} \vdash \bar{\alpha}_{Y}}}\left(\bigwedge \bar{\beta}_{X} \rightarrow \bigvee \bar{\beta}_{Y}\right), \\
& \beta_{n}:=\gamma_{n} \vee p_{n} \wedge \delta_{n} .
\end{aligned}
$$

Notice that it does not matter whether we read the definition of $\beta_{n}$ as $\gamma_{n} \vee\left(p_{n} \wedge \delta_{n}\right)$ or $\left(\gamma_{n} \vee p_{n}\right) \wedge \delta_{n}$, as $\vDash_{\text {CPC }} \gamma_{n} \rightarrow \delta_{n}$ : if $\bar{\alpha}_{X} \vdash \alpha_{n}, \bar{\alpha}_{Y}$ and $\bar{\alpha}_{W}, \alpha_{n} \vdash \bar{\alpha}_{Z}$, then $\bar{\alpha}_{X}, \bar{\alpha}_{W} \vdash \bar{\alpha}_{Y}, \bar{\alpha}_{Z}$, hence

$$
\vDash_{\mathbf{C P C}} \bigwedge \bar{\beta}_{X} \wedge \bigwedge \bar{\beta}_{W} \rightarrow \bigvee \bar{\beta}_{Y} \vee \bigvee \bar{\beta}_{Z}
$$

by (1), i.e.,

$$
\vDash_{\mathbf{C P C}}\left(\bigwedge \bar{\beta}_{X} \wedge \neg \bigvee \bar{\beta}_{Y}\right) \rightarrow\left(\bigwedge \bar{\beta}_{W} \rightarrow \bigvee \bar{\beta}_{Z}\right)
$$

We claim that (1) holds for $X, Y \subseteq n+1$. If $X, Y \subseteq n$, this follows from the induction hypothesis. If $n \in X \cap Y$, then trivially $\bar{\beta}_{X} \vDash_{\text {CPC }} \bar{\beta}_{Y}$. Assume that $\bar{\alpha}_{X} \vdash \alpha_{n}, \bar{\alpha}_{Y}$, where $X, Y \subseteq n$. Then

$$
\bigwedge \bar{\beta}_{X} \wedge \neg \bigvee \bar{\beta}_{Y} \vDash_{\mathbf{C P C}} \gamma_{n} \vDash_{\mathbf{C P C}} \beta_{n},
$$

hence by reasoning in $\mathbf{C P C}$,

$$
\bar{\beta}_{X} \vDash_{\mathbf{C P C}} \bar{\beta}_{Y}, \beta_{n}
$$

The case $\bar{\alpha}_{X}, \alpha_{n} \vdash \bar{\alpha}_{Y}$ is handled similarly using the definition of $\delta_{n}$.

Thus, $f$ is well defined, and by (1) and finitarity of $L$, it is a translation of $L$ to $\mathbf{C P C}_{m}$. In order to show that $f$ is conservative, assume that $\bar{\alpha}_{W} \nvdash \bar{\alpha}_{Z}$, we need to prove $\bar{\beta}_{W} \nvdash_{\mathbf{C P C}} \bar{\beta}_{Z}$. Obviously, $W \cap Z=\varnothing$. By the cut rule (i.e., Definition 2.5 (iii), applied with $\Xi=F$ ), we may assume that $W \cup Z=\omega$. Let $v$ be the valuation such that

$$
v\left(p_{n}\right)= \begin{cases}1, & n \in W, \\ 0, & n \in Z .\end{cases}
$$

We will show $v\left(\beta_{n}\right)=v\left(p_{n}\right)$ by induction on $n$, which implies $\bar{\beta}_{W} \nvdash_{\mathbf{C P C}} \bar{\beta}_{Z}$.

Assume that $n \in W$. If $X, Y \subseteq n$ are such that $\bar{\alpha}_{X}, \alpha_{n} \vdash \bar{\alpha}_{Y}$, then we cannot have simultaneously $X \subseteq W$ and $Y \subseteq Z$. If $i \in X \backslash W$, then $v\left(\beta_{i}\right)=0$ by the induction hypothesis; similarly, if $i \in Y \backslash Z$, then $v\left(\beta_{i}\right)=1$. Thus, $v\left(\bigwedge \bar{\beta}_{X}\right)=0$ or $v\left(\bigvee \bar{\beta}_{Y}\right)=1$. Since $X, Y$ were arbitrary, we obtain $v\left(\beta_{n}\right)=v\left(\delta_{n}\right)=1$.

If $n \in Z$, we obtain $v\left(\beta_{n}\right)=v\left(\gamma_{n}\right)=0$ by a similar argument.

Clearly, the explicit recursive definition of $f$ can be realized by an algorithm with an oracle for $\vdash$. On the other hand, since $f$ is a conservative translation into the decidable logic

\footnotetext{
${ }^{1}$ Note that the $\beta_{n}$ are defined by complete (ordinal) induction, which requires no separate base case. For example, the given definition implies that $\beta_{0}$ is one of $\top \vee p_{0} \wedge \top(\equiv \top), \perp \vee p_{0} \wedge \perp(\equiv \perp)$, or $\perp \vee p_{0} \wedge \top$ $\left(\equiv p_{0}\right)$, depending on whether $\varnothing \vdash \alpha_{0}, \alpha_{0} \vdash \varnothing$, or neither, respectively.
} 
CPC $\mathbf{C}_{m}$, the relation $\vdash$ is Turing reducible to $f$ (or its graph, if we insist on oracles being sets rather than functions).

It remains to show that $f$ is a most general translation of $L$ to $\mathbf{C P C}_{m}$. Let $g: L \rightarrow \mathbf{C P C}_{m}$, and let $\sigma$ be the substitution defined by $\sigma\left(p_{n}\right)=g\left(\alpha_{n}\right)$. We have to establish

$$
\vDash_{\text {CPC }} g\left(\alpha_{n}\right) \leftrightarrow \sigma\left(\beta_{n}\right)
$$

for every $n$, and we proceed by induction on $n$. The definition of $\beta_{n}$ implies that (2) is equivalent to

$$
\sigma\left(\gamma_{n}\right) \vDash_{\text {CPC }} g\left(\alpha_{n}\right) \vDash_{\text {CPC }} \sigma\left(\delta_{n}\right) .
$$

Using the definitions of $\gamma_{n}, \delta_{n}$ and the induction hypothesis, this is equivalent to

$$
\bigvee_{\substack{X, Y \subseteq n \\ \bar{\alpha}_{X} \vdash \alpha_{n}, \bar{\alpha}_{Y}}}\left(\bigwedge g\left(\bar{\alpha}_{X}\right) \wedge \neg \bigvee g\left(\bar{\alpha}_{Y}\right)\right) \vDash_{\mathbf{C P C}} g\left(\alpha_{n}\right) \vDash_{\mathbf{C P C}} \bigwedge_{\substack{X, Y \subseteq n \\ \bar{\alpha}_{X}, \alpha_{n} \vdash \bar{\alpha}_{Y}}}\left(\bigwedge g\left(\bar{\alpha}_{X}\right) \rightarrow \bigvee g\left(\bar{\alpha}_{Y}\right)\right),
$$

which in turn follows from the fact that $g$ is a translation: for example, if $X, Y \subseteq n$ are such that $\bar{\alpha}_{X} \vdash \alpha_{n}, \bar{\alpha}_{Y}$, then $g\left(\bar{\alpha}_{X}\right) \vDash_{\text {CPC }} g\left(\alpha_{n}\right), g\left(\bar{\alpha}_{Y}\right)$, hence $\wedge g\left(\bar{\alpha}_{X}\right) \wedge \neg \bigvee g\left(\bar{\alpha}_{Y}\right) \vDash_{\text {CPC }} g\left(\alpha_{n}\right)$.

Proof (of Theorem 2.4): Let $L=\langle F, \vdash\rangle$ be a finitary deductive system over countable $F$, and define its conservative multiple-conclusion extension $L^{m}=\left\langle F, \vdash^{m}\right\rangle$ by

$$
\Gamma \vdash^{m} \Delta \text { iff } \exists \psi \in \Delta \Gamma \vdash \psi .
$$

Let $f: L^{m} \rightarrow_{c} \mathbf{C P C}^{m}$ be the conservative minimal translation from Theorem 2.6. Since $\vdash_{\mathbf{C P C}}$ is the single-conclusion fragment of $\vDash_{\mathbf{C P C}}, f: L \rightarrow_{c}$ CPC. Moreover, if $g: L \rightarrow \mathbf{C P C}$, then $g: L^{m} \rightarrow \mathbf{C P C}_{m}$, hence $g$ is $\mathbf{C P C}$-equivalent to $\sigma \circ f$ for some substitution $\sigma$.

Remark 2.7 Even if $L=\mathbf{C P C}$, the translation $f$ from Theorem 2.4 is not (equivalent to) the identity, since it has the additional property that $f(\Gamma) \vdash_{\mathbf{C P C}} \bigvee_{i<n} f\left(\varphi_{i}\right)$ implies $f(\Gamma) \vdash_{\text {CPC }} f\left(\varphi_{i}\right)$ for some $i<n$.

Remark 2.8 Let us estimate the complexity of our translation $f$. Assume that formulas are represented by strings in a finite alphabet and enumerated in the natural way so that shorter formulas have smaller index, and consider a formula $\varphi$ of length $n$, so that $\varphi=\alpha_{m}$ for some $m=2^{O(n)}$. In order to compute $f(\varphi)$, we have to determine whether $\Gamma \vdash \Delta$ for sets $\Gamma, \Delta$ of formulas whose length is at most $n$; there are $2^{O(n)}$ such formulas, hence $2^{2^{O(n)}}$ sets. Thus, we define $f(\varphi)$ by iteration of length $2^{O(n)}$, and in each step, the formula $\beta_{i}$ is constructed from at most $2^{2^{O(n)}}$ copies of formulas constructed earlier. It follows that the total length of $f(\varphi)$ is $\left(2^{2^{O(n)}}\right)^{2^{O(n)}}=2^{2^{O(n)}}$.

Assume that $\vdash$ is decidable in $\operatorname{EXP}=\operatorname{DTIME}\left(2^{n^{O(1)}}\right)$. Since each of the sequents $\Gamma \vdash \Delta$ above has size $s=2^{O(n)}, f(\varphi)$ is computable in time $2^{2^{O(n)}} 2^{s^{O(1)}}=2^{2^{O(n)}}$. Moreover, if we compute $f$ in a left-to-right fashion by a recursive procedure mimicking its definition, we need recursion depth $2^{O(n)}$ and local storage $2^{O(n)}$ for each recursive call, plus the space needed to check $\Gamma \vdash \Delta$. Thus, if $\vdash \in \operatorname{PSPACE}$, then $f(\varphi)$ is computable in space $2^{O(n)}$.

In general, if $\vdash$ is computable in time $t(n)$ and space $s(n)$, where both $t$ and $s$ are monotone, then $f(\varphi)$ is computable in time $2^{2^{O(n)}} t\left(2^{O(n)}\right)$ and space $2^{O(n)}+s\left(2^{O(n)}\right)$. 


\section{Universal logics}

From now on, we only consider single-conclusion logics.

Definition 3.1 A deductive system $L_{0}$ is universal if $L \leq_{c} L_{0}$ for every finitary deductive system $L$ over countably many formulas.

(In all cases where we establish universality below, it is possible to construct an $f: L \rightarrow_{c} L_{0}$ Turing equivalent to $\vdash_{L}$, as in the case of $\mathbf{C P C}$. However, we decided not to include this condition in the definition.)

In the previous section, we proved that CPC is a universal logic. The main result of this section, Theorem 3.10, is a generalization of our construction to a large class of nonclassical logics in place of $\mathbf{C P C}$.

Before we get to the main result, we discuss some examples showing that the question of which logics CPC can be conservatively translated into is considerably more subtle than which logics can be conservatively translated into CPC.

From Theorem 2.4 and the transitivity of $\leq_{c}$, we immediately obtain:

\section{Observation 3.2}

(i) If $L_{0}$ is universal and $L_{0} \leq_{c} L_{1}$, then $L_{1}$ is universal.

(ii) $L_{0}$ is universal iff $\mathbf{C P C} \leq_{c} L_{0}$.

Example 3.3 Let $A_{3}=\langle\{0, *, 1\}, \wedge, \vee, 0,1, \neg\rangle$ be the 3 -element bounded lattice endowed with an operation $\neg 0=1, \neg 1=0, \neg *=*$. Recall that Kleene's 3-valued logic $\mathbf{K}$ (with truth constants) is the propositional logic using connectives $\wedge, \vee, \neg, \top, \perp$ whose consequence relation is defined by $A_{3}$ where 1 is the only designated value, and the paraconsistent logic of paradox $\mathbf{L P}$ is defined similarly but with both $1, *$ taken as designated. We have:

(i) $\mathbf{K}$ is universal.

(ii) $\mathbf{L P}$ is not universal.

(Notice that $\mathbf{K}$ without truth constants is trivially not universal, as it has no tautologies.)

Proof: (i): Let $f(\varphi)$ be a conjunctive normal form of $\varphi$, obeying the convention that no variable and its negation can appear simultaneously in a clause, and we use $\top, \perp$ for empty conjunctions and disjunctions, respectively. We claim that $f: \mathbf{C P C} \rightarrow_{c} \mathbf{K}$. Since $\mathbf{K} \subseteq \mathbf{C P C}$ and $\varphi \vdash_{\text {CPC }} f(\varphi)$, we clearly have

$$
f(\Gamma) \vdash_{\mathbf{K}} f(\varphi) \Rightarrow \Gamma \vdash_{\mathbf{C P C}} \varphi .
$$

In order to show the converse implication, it suffices to prove that

$$
\Gamma \vdash_{\mathbf{C P C}} \varphi \Rightarrow \Gamma \vdash_{\mathbf{K}} \varphi
$$


holds whenever $\Gamma \cup\{\varphi\}$ is a set of clauses. Let $v$ be a valuation in $A_{3}$ such that $v(\Gamma)=1$ and $v(\varphi) \neq 1$. We modify $v$ to make it a Boolean valuation $v^{\prime}$ as follows. If $l \in \varphi$ is a literal such that $v(l)=*$, we put $v^{\prime}(l)=0$; we can do this for all such $l$ simultaneously since $\varphi$ does not contain both $l$ and $\neg l$. If $p$ is a variable such that $v(p)=*$ and neither $p$ nor $\neg p$ appears in $\varphi$, we pick $v^{\prime}(p) \in\{0,1\}$ arbitrarily. After this modification, $v^{\prime}$ is a classical valuation such that $v^{\prime}(\varphi)=0$, and since all literals with value 1 kept their value, we still have $v^{\prime}(\Gamma)=1$.

(ii): Assume for contradiction $f: \mathbf{C P C} \rightarrow_{c} \mathbf{L P}$. Let $\left\{v_{i}: i<n\right\}$ be the list of all valuations in $A_{3}$ such that $v_{i}(f(\perp))=0$ with $v_{i}\left(p_{j}\right)=*$ for every variable $p_{j}$ not occurring in $f(\perp)$. Put $\varphi_{i}=p_{i}$ for $i<n, \varphi_{n}=\neg \bigwedge_{i<n} p_{i}$. We have $\varphi_{0}, \ldots, \varphi_{n} \vdash_{\text {CPC }} \perp$, which implies $f\left(\varphi_{0}\right), \ldots, f\left(\varphi_{n}\right) \vdash_{\mathbf{L P}} f(\perp)$. Since $v_{i}(f(\perp))=0$, we must have $v_{i}\left(f\left(\varphi_{j_{i}}\right)\right)=0$ for some $j_{i} \leq n$. Put $J=\left\{j_{i}: i<n\right\}$. We claim that

$$
\left\{f\left(\varphi_{j}\right): j \in J\right\} \vdash_{\mathbf{L P}} f(\perp)
$$

Indeed, if $v(f(\perp))=0$, there exists an $i$ such that $v$ and $v_{i}$ coincide on variables occurring in $f(\perp)$. We have $v_{i}\left(f\left(\varphi_{j}\right)\right)=0$ for some $j \in J$. If $\prec$ is the partial order induced by $* \prec 0$, $* \prec 1$, then functions definable in $A_{3}$ are $\prec$-monotone, and $v_{i} \preceq v$, hence $v\left(f\left(\varphi_{j}\right)\right)=0$. Thus, by the conservativity of $f$,

$$
\left\{\varphi_{j}: j \in J\right\} \vdash_{\mathbf{C P C}} \perp \text { for some }|J| \leq n .
$$

This contradicts the definition of $\varphi_{0}, \ldots, \varphi_{n}$.

In order to get some insight which logics can or cannot be expected to be universal depending on their available list of connectives, we characterize universal fragments of classical logic below.

Definition 3.4 If $L$ is a propositional logic, and $B$ a set of connectives definable in $L$ (i.e., $L$-formulas), we denote by $L \uparrow_{B}$ the fragment of $L$ using only formulas built from $B$. (We treat $L \uparrow_{B}$ as having $B$ as the basic set of connectives, regardless of the basic connectives of $L$.)

A clone on a set $X$ is a set of finitary operations on $X$ which is closed under composition and contains all projections. If $B$ is a set of operations on $X$, then we denote by $[B]$ the clone generated by $B$. Notice that if $B$ is a set of Boolean functions, then definable functions in $\left.\mathbf{C P C}\right|_{B}$ are exactly the functions from $[B]$, hence clones on $\{0,1\}$ are in $1-1$ correspondence with fragments of $\mathbf{C P C}$ considered up to term equivalence.

The lattice of clones on $\{0,1\}$ was completely described by Post [11] (see also Lau [9] for a modern exposition). We will in particular need to refer to the following clones:

- The clone $P_{0}$ of all 0-preserving functions (i.e., $\left.f(0, \ldots, 0)=0\right)$.

- The clone $D$ of all self-dual functions (i.e., $f\left(\neg x_{1}, \ldots, \neg x_{n}\right)=\neg f\left(x_{1}, \ldots, x_{n}\right)$ ).

- The clone $A$ of all affine functions $\left(f\left(x_{1}, \ldots, x_{n}\right)=\sum_{i \in I} x_{i}+c\right.$, where $c \in\{0,1\}$, $I \subseteq\{1, \ldots, n\}$, and + denotes addition modulo 2$)$.

- The clone $M$ of all monotone functions. 
- The clone $T_{1}^{\infty}$ of functions bounded below by a variable (there exists $i$ such that $x_{i} \leq$ $f\left(x_{1}, \ldots, x_{n}\right)$ for every $\left.\vec{x} \in\{0,1\}^{n}\right)$.

The following lemma follows immediately from inspection of Post's lattice, though we invite the reader to give a direct proof:

Lemma 3.5 If $B$ is a set of Boolean functions, then $\rightarrow \notin[B]$ if and only if $B$ is included in $P_{0}, D, A$, or $M$.

Theorem 3.6 Let $B$ be a set of Boolean functions. The fragment $\mathbf{C P C} \uparrow_{B}$ is universal if and only if $\rightarrow$ is definable from $B$.

Proof: Left-to-right: if $\rightarrow \notin[B]$, then $B$ is included in one of the clones mentioned in Lemma 3.5. If $B \subseteq P_{0}$ or $B \subseteq D$, then $\top \notin[B]$; in other words, $\left.\mathbf{C P C}\right|_{B}$ has no tautologies, and therefore cannot be universal.

Let $B \subseteq A$. We claim that if $\varphi, \psi \in A$, then $\varphi \leq \psi$ only if $\varphi=0$ or $\psi=1$ or $\varphi=\psi$. Write $\varphi(\vec{x})=\sum_{i \in I} x_{i}+c, \psi(\vec{x})=\sum_{i \in J} x_{i}+d$. If $\psi \neq 1$, there is a Boolean valuation $v$ such that $v(\psi)=0$. If $I \nsubseteq J$, we can change the valuation of any $x_{i}$ such that $i \in I \backslash J$ to make $v(\varphi)=1$, contradicting $\varphi \leq \psi$. Thus, $\psi=1$ or $I \subseteq J$. Since $\varphi \leq \psi$ implies $\neg \psi \leq \neg \varphi$, the same argument gives $\varphi=0$ or $J \subseteq I$. Finally, if $I=J$, then $\psi=\varphi$ or $\psi=\neg \varphi$; in the latter case, $\varphi \leq \psi$ can only hold if $\varphi$ and $\psi$ are constant functions 0 and 1 , respectively.

In particular, there is no strictly increasing chain of length more than 3 of affine functions ordered by entailment, hence we cannot conservatively translate CPC (even with just 2 variables) into $\mathbf{C P C} \uparrow_{B}$.

If $B \subseteq M$, we will show $\left.\mathbf{C P C}\right|_{B} \leq{ }_{c} \mathbf{L P}$, hence $\left.\mathbf{C P C}\right|_{B}$ is not universal by Example 3.3. Since $M=[\wedge, \vee, \top, \perp]$, we may assume $B=\{\wedge, \vee, \top, \perp\}$. Let $\sigma$ be the substitution such that $\sigma(p)=p \wedge \neg p$. We claim

$$
\Gamma \vdash_{\mathbf{C P C}_{B}} \varphi \Leftrightarrow \sigma(\Gamma) \vdash_{\mathbf{L P}} \sigma(\varphi)
$$

Notice that $\sigma$, being a substitution, is a bounded lattice homomorphism of the respective free algebras. Let $A_{2}$ denote the 2-element bounded lattice. The mapping $\pi: A_{3} \rightarrow A_{2}$ such that $\pi(1)=\pi(*)=1, \pi(0)=0$, is also a bounded lattice homomorphism, and it preserves (in both directions) the sets of designated elements.

If $v$ is a valuation in $A_{3}$ such that $v(\sigma(\Gamma)) \geq *, v(\sigma(\varphi))=0$, then $v^{\prime}=\pi \circ v \circ \sigma$ is a valuation in $A_{2}$ such that $v^{\prime}(\Gamma)=1$ and $v^{\prime}(\varphi)=0$, hence $\Gamma \nvdash_{\mathbf{C P C}} \varphi$.

Conversely, if $v^{\prime}$ is a valuation in $A_{2}$ such that $v^{\prime}(\Gamma)=1$ and $v^{\prime}(\varphi)=0$, let $v$ be the valuation in $A_{3}$ induced by

$$
v\left(p_{i}\right)= \begin{cases}* & v^{\prime}\left(p_{i}\right)=1, \\ 0 & v^{\prime}\left(p_{i}\right)=0 .\end{cases}
$$

Then $\pi \circ v \circ \sigma=v^{\prime}$, hence $v(\sigma(\Gamma)) \geq *, v(\sigma(\varphi))=0$.

Right-to-left: we construct $f: \mathbf{C P C} \rightarrow \mathbf{C P C} \uparrow \rightarrow$ as follows. First, we rename all propositional variables in the style of Hilbert's hotel so that we obtain a spare variable $q$ which does 
not occur in any formulas. Then, for each formula $\varphi$ not containing $q$, let $f(\varphi)$ be an implicational formula equivalent to $\varphi \vee q$; it exists as $[\rightarrow]=T_{1}^{\infty}$. (For a more explicit construction, we can use the functional completeness of $\{\rightarrow, \perp\}$ to write $\varphi(\vec{p}) \leftrightarrow \psi(\vec{p}, \perp)$ for some $\psi \in[\rightarrow]$, and then put $f(\varphi)=(\psi(\vec{p}, q) \rightarrow q) \rightarrow q$.) It is easy to see that $f: \mathbf{C P C} \rightarrow_{c} \mathbf{C P C} \uparrow \rightarrow$.

Remark 3.7 Ideally, we would like to prove that a logic is universal whenever it meets some simple general conditions, such as those studied in abstract algebraic logic (see [2]). However, on the one hand, the affine fragments $\left.\mathbf{C P C}\right|_{\leftrightarrow}$ or $\left.\mathbf{C P C}\right|_{\leftrightarrow, \neg}$ are strongly regularly finitely algebraizable (i.e., as nice as it can get from the point of view of AAL), on the other hand, Kleene's logic is not even equivalential. This shows that universality does not have much to do with abstract algebraic properties of the logic. Consequently, if we want to establish universality of a class of logics, we cannot rely only on their general properties, at some point we have to resort to working with particular systems. We at least try to pick as weak a base system as possible so that our result covers a broad class of logics including most systems studied in the literature.

Definition 3.8 A residuated lattice is a structure $\langle L, \wedge, \vee, \cdot, \rightarrow, \leftarrow, 1\rangle$ where $\langle L, \wedge, \vee\rangle$ is a lattice, $\langle L, \cdot, 1\rangle$ is a monoid, and

$$
b \leq a \rightarrow c \Leftrightarrow a \cdot b \leq c \Leftrightarrow a \leq c \leftarrow b
$$

for every $a, b, c \in L$. (In particular, $a \cdot(a \rightarrow b) \leq b,(b \leftarrow a) \cdot a \leq b)$. An FL-algebra is a residuated lattice $L$ with a distinguished point $0 \in L$. The full Lambek calculus FL is the propositional logic using connectives $\wedge, \vee, \cdot, \rightarrow, \leftarrow, 1,0$ such that $\vdash_{\text {FL }}$ is complete with respect to the class of logical matrices whose underlying algebras are FL-algebras $L$, with $\{x \in L: x \geq 1\}$ taken as the set of designated elements. $\mathbf{F L}_{\mathbf{e}}$ is complete with respect to commutative FL-algebras $(x \cdot y=y \cdot x)$, and $\mathbf{F L}_{\text {ew }}$ with respect to 0-bounded integral $(0 \leq x \leq 1)$ commutative FL-algebras. In a sequent calculus formulation of $\mathbf{F L}$, e corresponds to the exchange rule, and $\mathbf{w}$ to the weakening rule. For more information about FL and its extensions or fragments, we refer the reader to [8].

If $\Gamma=\left\langle\varphi_{1}, \ldots, \varphi_{k}\right\rangle$ is a sequence of formulas, we define

$$
\begin{aligned}
& \Gamma \rightarrow \psi:=\varphi_{1} \rightarrow\left(\varphi_{2} \rightarrow\left(\varphi_{3} \rightarrow \cdots\left(\varphi_{k} \rightarrow \psi\right) \cdots\right)\right), \\
& \psi \leftarrow \Gamma:=\left(\cdots\left(\left(\psi \leftarrow \varphi_{1}\right) \leftarrow \varphi_{2}\right) \cdots \leftarrow \varphi_{k-1}\right) \leftarrow \varphi_{k} .
\end{aligned}
$$

If $k=0$, it is understood that $\Gamma \rightarrow \psi=\psi \leftarrow \Gamma=\psi$. We also put $\prod \Gamma=\varphi_{1} \cdot \varphi_{2} \cdot \ldots \cdot \varphi_{k}$ $\left(\prod \Gamma=1\right.$ if $\left.k=0\right)$, and $\varphi^{k}=\underbrace{\varphi \cdot \varphi \cdot \ldots \cdot \varphi}_{k \text { times }}$. Notice that in $\mathbf{F L}, \Gamma \rightarrow \psi$ is equivalent (in the sense of obtaining the same value under any valuation in any FL-algebra) to $\prod \Gamma^{-1} \rightarrow \psi$, and $\psi \leftarrow \Gamma$ is equivalent to $\psi \leftarrow \Pi \Gamma^{-1}$, where $\Gamma^{-1}$ denotes the reversal of the sequence $\Gamma$.

Remark 3.9 Let $L$ be a finitary deductive system over countably many formulas $F=\left\{\alpha_{n}\right.$ : $n<\omega\}$. From the proofs of Theorems 2.4 and 2.6 we know that there is a conservative 
translation $f: L \rightarrow_{c}$ CPC such that $f\left(\alpha_{n}\right)=\beta_{n}$ is inductively defined to be equivalent to the formula

$$
\bigwedge_{\substack{X \subseteq n>k \\ \bar{\alpha}_{X}, \alpha_{n} \vdash_{L} \alpha_{k}}}\left(\bar{\beta}_{X} \rightarrow \beta_{k}\right) \wedge\left(p_{n} \vee \bigvee_{\substack{Z \subseteq n \\ \bar{\alpha}_{Z} \vdash L}} \bigwedge \bar{\beta}_{Z}\right)
$$

Theorem 3.10 A deductive system is universal whenever it conservatively extends a deductive system $L_{0}$ such that

(i) $\mathbf{F L} \uparrow \rightarrow, \leftarrow, \wedge \subseteq L_{0} \subseteq \mathbf{C P C} \uparrow_{\rightarrow, \leftarrow, \wedge}$ or

(ii) $\mathbf{F L}_{\mathbf{e}} \uparrow_{\rightarrow, \wedge} \subseteq L_{0} \subseteq \mathbf{C P C} \uparrow_{\rightarrow, \wedge}$, or

(iii) $\mathbf{F L}_{\mathbf{e w}} \uparrow \rightarrow \mathbf{B C K} \subseteq L_{0} \subseteq \mathbf{C P C} \uparrow \rightarrow \cdot$

Proof: (i): Let $L$ be as in Remark 3.9, we will show $L \leq_{c} L_{0}$. Put $\pi(p, q)=(p \rightarrow q) \rightarrow q$. Using the notation from Definition 3.8, Remark 3.9, and from the proof of Theorem 2.6, we put $f\left(\alpha_{n}\right)=\beta_{n}$, where we define inductively

$$
\left.\beta_{n}:=(q \rightarrow q) \wedge \bigwedge_{\vec{\alpha}_{X}, \vec{\alpha}_{Y}, \alpha_{n} \vdash_{L} \alpha_{k}}\left(\left(\vec{\beta}_{Y} \rightarrow \beta_{k}\right) \leftarrow \vec{\beta}_{X}\right) \wedge\left(\bigwedge_{\vec{\alpha}_{Z} \vdash_{L} \alpha_{n}}\left(\pi\left(p_{n}, q\right) \leftarrow \vec{\beta}_{Z}\right)\right) \rightarrow \pi\left(p_{n}, q\right)\right) .
$$

The first big conjunction in $\beta_{n}$ is taken over all $k<n$ and all repetition-free disjoint sequences $X$ and $Y$ consisting of elements $i<n$ such that $\vec{\alpha}_{X}, \vec{\alpha}_{Y}, \alpha_{n} \vdash_{L} \alpha_{k}$, and similarly for the second conjunction. (Here, if $X=\left\langle i_{1}, \ldots, i_{m}\right\rangle$, we define $\vec{\alpha}_{X}$ to be the sequence $\left\langle\alpha_{i_{1}}, \ldots, \alpha_{i_{m}}\right\rangle$, and similarly for $\vec{\beta}_{X}$.) If there are no $Z \subseteq n$ such that $\vec{\alpha}_{Z} \vdash_{L} \alpha_{n}$, then the last conjunct of $\beta_{n}$ is understood to be just $\pi\left(p_{n}, q\right)$.

Since $\beta_{n}(q / \perp)$ is classically equivalent to (3), we obtain immediately

$$
f(\Gamma) \vdash_{L_{0}} f(\varphi) \Rightarrow f(\Gamma) \vdash_{\mathbf{C P C}} f(\varphi) \Rightarrow \Gamma \vdash_{L} \varphi
$$

In order to show

$$
\Gamma \vdash_{L} \varphi \Rightarrow f(\Gamma) \vdash_{\mathbf{F L}} f(\varphi) \Rightarrow f(\Gamma) \vdash_{L_{0}} f(\varphi),
$$

it suffices to prove by induction on $n$ that for every $k<n$ and every sequence $Z$ of elements of $n$,

$$
\vec{\alpha}_{Z} \vdash_{L} \alpha_{k} \Rightarrow \vdash_{\mathbf{F L}} \vec{\beta}_{Z} \rightarrow \beta_{k}
$$

(then $\vec{\beta}_{Z} \vdash_{\text {FL }} \beta_{k}$ by modus ponens). The statement is vacuously true for $n=0$. Assume that it holds for $n$, we will prove it for $n+1$.

Claim 1 Let $\langle L, \wedge, \vee, \cdot, \rightarrow, \leftarrow, 1\rangle$ be a residuated lattice, and $u \in L$.

(i) $L_{u}:=\{a \in L: a u, u a \leq a\}$ is closed under $\rightarrow, \leftarrow, \wedge$ (as well as $\cdot, \vee$, but we will not need this).

(ii) If $u=q \rightarrow q$ for some $q \in L$, then $1 \leq u, u^{2} \leq u$, and $L_{u}$ contains $u$ as well as all elements of the form $\pi(a, q)$. 
(iii) FL proves $\beta_{i} \cdot \beta_{j} \rightarrow \beta_{i}, \beta_{j} \cdot \beta_{i} \rightarrow \beta_{i}$, and $\vec{\beta}_{X} \rightarrow(q \rightarrow q)$.

Proof: (i): Let $a, b \in L_{u}$.

We have $u(a \wedge b) \leq u a \leq a$ and $u(a \wedge b) \leq u b \leq b$, hence $u(a \wedge b) \leq a \wedge b$. The proof of $(a \wedge b) u \leq a \wedge b$ is symmetric.

Since $a(a \rightarrow b) u \leq b u \leq b$, we have $(a \rightarrow b) u \leq a \rightarrow b$. Similarly, $a u(a \rightarrow b) \leq a(a \rightarrow b) \leq$ $b$, hence $u(a \rightarrow b) \leq a \rightarrow b$.

The case of $a \leftarrow b$ is symmetric.

(ii): $1 \leq u$ is clear, and $u^{2} \leq u$ (which implies $u \in L_{u}$ ) is a special case of

$$
(a \rightarrow b)(b \rightarrow c) \leq a \rightarrow c .
$$

Put $p=(a \rightarrow q) \rightarrow q$. We have $p(q \rightarrow q) \leq p$ from $(5)$. Also, $(a \rightarrow q)(q \rightarrow q) p \leq(a \rightarrow q) p \leq$ $q$, hence $(q \rightarrow q) p \leq p$.

(iii): Consider a valuation $v$ in a residuated lattice $L$, and put $u=v(q) \rightarrow v(q)$. Notice that $\beta_{i}$ is ultimately constructed from formulas of the form $\pi\left(p_{k}, q\right)$ and $q \rightarrow q$ by means of $\rightarrow, \leftarrow, \wedge$, thus $v\left(\beta_{i}\right) \in L_{u}$ by (i) and (ii). Clearly, $v\left(\beta_{j}\right) \leq u$, hence $v\left(\beta_{i}\right) v\left(\beta_{j}\right) \leq v\left(\beta_{i}\right)$ and $v\left(\beta_{j}\right) v\left(\beta_{i}\right) \leq v\left(\beta_{i}\right)$. Finally, $v\left(\prod \vec{\beta}_{X^{-1}}\right) \leq u^{|X|} \leq u$ by (ii).

(Claim 1)

It follows from the claim that it is enough to prove (4) for repetition-free sequences $Z$ not containing $k$. The only interesting cases are those involving $n$ : i.e., $Z=X \frown n \frown Y$ or $k=n$.

Assume that $\vec{\alpha}_{X}, \alpha_{n}, \vec{\alpha}_{Y} \vdash_{L} \alpha_{k}$. Then the definition of $\beta_{n}$ ensures $\vdash_{\text {FL }} \beta_{n} \rightarrow\left(\left(\vec{\beta}_{Y} \rightarrow\right.\right.$ $\left.\left.\beta_{k}\right) \leftarrow \vec{\beta}_{X}\right)$, hence $\vdash_{\text {FL }} \beta_{n} \cdot \prod \vec{\beta}_{X^{-1}} \rightarrow\left(\vec{\beta}_{Y} \rightarrow \beta_{k}\right)$, which in turn gives $\vdash_{\text {FL }} \prod \vec{\beta}_{X^{-1}} \rightarrow\left(\beta_{n} \rightarrow\right.$ $\left.\left(\vec{\beta}_{Y} \rightarrow \beta_{k}\right)\right)$ and $\vdash_{\mathbf{F L}} \vec{\beta}_{X} \rightarrow\left(\beta_{n} \rightarrow\left(\vec{\beta}_{Y} \rightarrow \beta_{k}\right)\right)$.

Assume that $\vec{\alpha}_{Z} \vdash_{L} \alpha_{n}$. We have $\vdash_{\mathbf{F L}} \vec{\beta}_{Z} \rightarrow(q \rightarrow q)$ by the Claim. Whenever $\vec{\alpha}_{X}, \alpha_{n}, \vec{\alpha}_{Y} \vdash_{L} \alpha_{k}$, we have $\vec{\alpha}_{X}, \vec{\alpha}_{Z}, \vec{\alpha}_{Y} \vdash_{L} \alpha_{k}$ by cut, hence $\vdash_{\text {FL }} \vec{\beta}_{X} \rightarrow\left(\vec{\beta}_{Z} \rightarrow\left(\vec{\beta}_{Y} \rightarrow\right.\right.$ $\left.\left.\beta_{k}\right)\right)$ by the induction hypothesis. By a similar argument as above, this is equivalent to $\vdash_{\text {FL }} \vec{\beta}_{Z} \rightarrow\left(\left(\vec{\beta}_{Y} \rightarrow \beta_{k}\right) \leftarrow \vec{\beta}_{X}\right)$. Finally, that $\vec{\beta}_{Z}$ implies the last conjunct of $\beta_{n}$ follows from $\vdash_{\mathbf{F L}} \vec{\beta}_{Z} \rightarrow\left(\left(\pi\left(p_{n}, q\right) \leftarrow \vec{\beta}_{Z}\right) \rightarrow \pi\left(p_{n}, q\right)\right)$.

(ii) follows immediately from (i), as $(\varphi \leftarrow \psi)=(\psi \rightarrow \varphi)$ in $\mathbf{F L}_{\mathbf{e}}$.

(iii): We define inductively

$$
\begin{aligned}
r_{0} & :=0, \\
r_{n+1} & :=1+n 2^{n} r_{n}, \\
\varepsilon_{n} & :=\left(\prod_{\bar{\alpha}_{X}, \alpha_{n} \vdash_{L} \alpha_{k}}\left(\bar{\beta}_{X}^{r_{n}} \rightarrow \beta_{k}\right)\right) \cdot\left(\left(\prod_{\bar{\alpha}_{Z} \vdash_{L} \alpha_{n}}\left(\bar{\beta}_{Z} \rightarrow p_{n}\right)\right) \rightarrow p_{n}\right), \\
\beta_{n} & :=\left(\varepsilon_{n} \rightarrow q\right) \rightarrow q,
\end{aligned}
$$

where the products are taken over $X, Z \subseteq n, k<n$. (Unlike the case of $\mathbf{F L}$, we can treat here $X, Z$ as sets, because fusion is commutative.) We understand $\bar{\beta}_{X}^{r_{n}}$ to be the multiset of formulas which contains $r_{n}$ copies of each formula $\beta_{i}, i \in X$ (again, the order does not matter due to commutativity). That is, if $X=\left\{i_{1}, \ldots, i_{m}\right\}$ (in an arbitrary order), then $\bar{\beta}_{X}^{r_{n}} \rightarrow \beta_{k}$ stands for

$$
\underbrace{\beta_{i_{1}} \rightarrow\left(\beta _ { i _ { 1 } } \cdots \rightarrow \left(\beta_{i_{1}}\right.\right.}_{r_{n}} \rightarrow(\underbrace{\beta_{i_{2}} \cdots \rightarrow\left(\beta_{i_{2}}\right.}_{r_{n}} \rightarrow\left(\cdots \rightarrow(\underbrace{\beta_{i_{m}} \cdots \rightarrow\left(\beta_{i_{m}}\right.}_{r_{n}} \rightarrow \beta_{k})\right)) \cdots))) .
$$


Notice that fusion only appears in $\beta_{n}$ in premises of implications, hence $\beta_{n}$ can be equivalently rewritten as a formula $f\left(\alpha_{n}\right)$ using only $\rightarrow$.

Since $\beta_{n}(q / \perp)$ is classically equivalent to $(3)$, we have

$$
f(\Gamma) \vdash_{L_{0}} f(\varphi) \Rightarrow f(\Gamma) \vdash_{\mathbf{C P C}} f(\varphi) \Rightarrow \Gamma \vdash_{L} \varphi
$$

By induction on $n$, we will show that

$$
\bar{\alpha}_{W} \vdash_{L} \alpha_{k} \Rightarrow \vdash_{\mathbf{F L}_{\mathbf{e w}}} \bar{\beta}_{W}^{r_{n}} \rightarrow \beta_{k}
$$

holds for every $k<n$ and every $W \subseteq n$, which implies

$$
\Gamma \vdash_{L} \varphi \Rightarrow f(\Gamma) \vdash_{\mathbf{F L}_{\text {ew }}} f(\varphi) \Rightarrow f(\Gamma) \vdash_{L_{0}} f(\varphi) .
$$

The statement is vacuously true for $n=0$. Assume that it holds for $n$, we will prove it for $n+1$. Since we have weakening, it suffices to consider the cases $k=n, W \subseteq n$ and $k<n$, $W=X \cup\{n\}, X \subseteq n$.

Assume $\bar{\alpha}_{X}, \alpha_{n} \vdash_{L} \alpha_{k}$. Using the definition and commutativity, we have $\vdash_{\mathbf{F L}_{\mathbf{e w}}} \bar{\beta}_{X}^{r_{n}} \rightarrow$ $\left(\varepsilon_{n} \rightarrow \beta_{k}\right)$, which implies

$$
\vdash_{\mathbf{F L}_{\text {ew }}} \bar{\beta}_{X}^{r_{n}} \rightarrow\left(\left(\beta_{k} \rightarrow q\right) \rightarrow\left(\varepsilon_{n} \rightarrow q\right)\right) .
$$

The definition of $\beta_{k}$ gives $\vdash_{\mathbf{F L}_{\mathbf{e w}}}\left(\varepsilon_{k} \rightarrow q\right) \rightarrow\left(\beta_{k} \rightarrow q\right)$ using commutativity, hence

$$
\vdash_{\mathbf{F L}_{\mathrm{ew}}} \bar{\beta}_{X}^{r_{n}} \rightarrow\left(\left(\varepsilon_{k} \rightarrow q\right) \rightarrow\left(\varepsilon_{n} \rightarrow q\right)\right) .
$$

This implies $\vdash_{\mathbf{F L}_{\mathbf{e w}}} \bar{\beta}_{X}^{r_{n}} \rightarrow\left(\left(\left(\varepsilon_{n} \rightarrow q\right) \rightarrow q\right) \rightarrow\left(\left(\varepsilon_{k} \rightarrow q\right) \rightarrow q\right)\right)$, i.e., $\vdash_{\mathbf{F L}_{\mathbf{e w}}} \bar{\beta}_{X}^{r_{n}} \rightarrow\left(\beta_{n} \rightarrow \beta_{k}\right)$. We obtain

$$
\vdash_{\mathbf{F L}_{\text {ew }}} \bar{\beta}_{X}^{r_{n+1}} \rightarrow\left(\beta_{n}^{r_{n+1}} \rightarrow \beta_{k}\right)
$$

by weakening, using $r_{n} \leq r_{n+1}$.

Assume $\bar{\alpha}_{W} \vdash_{L} \alpha_{n}$. We have $\vdash_{\mathbf{F L}_{\mathbf{e w}}} \bar{\beta}_{W} \rightarrow\left(\left(\bar{\beta}_{W} \rightarrow p_{n}\right) \rightarrow p_{n}\right)$, hence

$$
\vdash_{\mathbf{F L}_{\text {ew }}} \bar{\beta}_{W} \rightarrow\left(\left(\prod_{\bar{\alpha}_{Z} \vdash_{L} \alpha_{n}}\left(\bar{\beta}_{Z} \rightarrow p_{n}\right)\right) \rightarrow p_{n}\right)
$$

by weakening. Whenever $\bar{\alpha}_{X}, \alpha_{n} \vdash_{L} \alpha_{k}$, we have $\bar{\alpha}_{X}, \bar{\alpha}_{W} \vdash_{L} \alpha_{k}$ by cut, hence

$$
\vdash_{\mathbf{F L}} \bar{\beta}_{W}^{r_{n}} \rightarrow\left(\bar{\beta}_{X}^{r_{n}} \rightarrow \beta_{k}\right)
$$

by the induction hypothesis and weakening. Since there are at most $n 2^{n}$ pairs $\langle X, k\rangle$ such that $X \subseteq n, k<n$, and $\bar{\alpha}_{X}, \alpha_{n} \vdash_{L} \alpha_{k}$, we have

$$
\vdash_{\mathbf{F L}} \bar{\beta}_{W}^{n 2^{n} r_{n}} \rightarrow \prod_{\bar{\alpha}_{X}, \alpha_{n} \vdash_{L} \alpha_{k}}\left(\bar{\beta}_{X}^{r_{n}} \rightarrow \beta_{k}\right) .
$$

Putting the pieces together, we have $\vdash_{\mathbf{F L}} \bar{\beta}_{W}^{r_{n+1}} \rightarrow \varepsilon_{n}$, hence

$$
\vdash_{\mathbf{F L}} \bar{\beta}_{W}^{r_{n+1}} \rightarrow \beta_{n}
$$


Remark 3.11 Every consistent substitution-invariant extension of BCK (in the same language) is contained in $\left.\mathbf{C P C}\right|_{\rightarrow}$. This is no longer true for $\left.\mathbf{F} \mathbf{L}_{\mathbf{e}}\right|_{\rightarrow, \wedge}$, nevertheless one can modify the proof above to show that (i) and (ii) of Theorem 3.10 remain true when CPC is replaced with any consistent substitution-invariant extension of $\mathbf{F L} \uparrow \rightarrow, \leftarrow, \wedge$ or $\mathbf{F L}_{\mathbf{e}} \uparrow_{\rightarrow, \wedge}$, respectively. We omit the details.

\section{Conclusion}

Our results (Theorems 2.4 and 3.10 and Example 3.3) show that any countable finitary deductive system can be conservatively translated into (among others):

- Classical propositional logic.

- Intuitionistic, minimal, and intermediate logics.

- Modal logics (classical or intuitionistic), including variants such as temporal or epistemic logics.

- Substructural logics, such as various extensions of FL or linear logic.

- Fuzzy and many-valued logics, such as MTL, BL and their extensions (e.g., Łukasiewicz logic).

- Relevant logics, such as R.

- Kleene's logic.

- First-order (or higher-order) extensions of the above logics.

- Implication fragments of many of the above logics.

This includes most of logical systems (fitting into the framework of Tarski-style consequence relations) studied in the literature on non-classical logic. We have also discovered some counterexamples, namely CPC cannot be conservatively translated into its monotone or affine fragments, or into the paraconsistent logic $\mathbf{L P}$.

While there are still some loose ends left (most importantly, we were unable to determine whether the logic $\mathbf{B C I}=\mathbf{F L}_{\mathbf{e}} \uparrow \rightarrow$ is universal, though it seems plausible), these results show that the mere existence of a conservative translation of one logic into another without further restrictions does not provide useful information on the relationship of the two logics, and a more refined criterion is needed to formalize the intuitive notion of translatability.

\section{Acknowledgements}

I would like to thank Petr Cintula for various helpful suggestions on a preliminary version of this paper, and the anonymous referees for useful comments. 


\section{References}

[1] Walter A. Carnielli, Marcelo E. Coniglio, and Itala M. Loffredo D'Ottaviano, New dimensions on translations between logics, Logica Universalis 3 (2009), no. 1, pp. 1-18.

[2] Janusz Czelakowski, Protoalgebraic logic, Trends in Logic vol. 10, Kluwer, 2001.

[3] Itala M. Loffredo D'Ottaviano and Hércules A. Feitosa, Many-valued logics and translations, Journal of Applied Non-classical Logics 9 (1999), no. 1, pp. 121-140.

[4] _ Paraconsistent logics and translations, Synthese 125 (2000), no. 1-2, pp. $77-$ 95.

[5] - Translating from Eukasiewicz's logics into classical logic: is it possible?, Poznan Studies in the Philosophy of the Sciences and the Humanities 91 (2006), no. 1, pp. $157-168$.

[6] - Deductive systems and translations, in: Perspectives on Universal Logic (J.-Y. Béziau and A. Costa-Leite, eds.), Polimetrica, 2007, pp. 125-157.

[7] Hércules A. Feitosa and Itala M. Loffredo D'Ottaviano, Conservative translations, Annals of Pure and Applied Logic 108 (2001), pp. 205-227.

[8] Nikolaos Galatos, Peter Jipsen, Tomasz Kowalski, and Hiroakira Ono, Residuated lattices: An algebraic glimpse at substructural logics, Studies in Logic and the Foundations of Mathematics vol. 151, Elsevier, Amsterdam, 2007.

[9] Dietlinde Lau, Function algebras on finite sets: A basic course on many-valued logic and clone theory, Springer, New York, 2006.

[10] Till Mossakowski, Răzvan Diaconescu, and Andrzej Tarlecki, What is a logic translation?, Logica Universalis 3 (2009), no. 1, pp. 95-124.

[11] Emil L. Post, The two-valued iterative systems of mathematical logic, Annals of Mathematics Studies no. 5, Princeton University Press, Princeton, 1941.

[12] D. J. Shoesmith and Timothy J. Smiley, Multiple-conclusion logic, Cambridge University Press, 1978.

[13] Jairo J. da Silva, Itala M. Loffredo D'Ottaviano, and Antônio M. Sette, Translations between logics, in: Models, Algebras, and Proofs (X. Caicedo and C. Montenegro, eds.), Lecture Notes in Pure and Applied Mathematics vol. 203, Marcel Dekker, New York, 1999, pp. 435-448. 